\title{
Liderazgo corporativo en tiempos de crisis económica: estudio de casos en empresas puertorriqueñas
}

\author{
Corporate leadership in times of economic crisis: \\ case study in Puerto Rican business
}

\section{Liderança corporativa em tempos de crise económica: estudo de caso em empresas de Puerto Rico}

\author{
Ph. D. Zaida I. Berdecía Cruz* \\ Ph. D. Joyce R. González Domínguez**
}

\section{RESUMEN}

El objetivo del estudio es identificar los rasgos y roles más prevalecientes en los líderes de empresas puertorriqueñas en momentos de crisis financiera. Mediante un enfoque cualitativo descriptivo, con diseño de estudio de caso múltiple, los resultados muestran que el líder eficiente es aquel que consigue mover al grupo a la consecución de las metas. Entre los rasgos más sobresalientes se encuentran: la honestidad, la empatía, el optimismo, la proactividad y la creatividad. Estos rasgos guardan relación con el estilo de liderazgo transformacional, necesario ante situaciones asociadas con la crisis financiera que impacta las empresas puertorriqueñas.

Palabras clave: liderazgo, rasgos del líder, roles del líder, crisis económica, Puerto Rico.

\begin{abstract}
The aim of the study is to identify the most prevalent traits and roles in the leaders of Puerto Rican companies in times of financial crisis. A descriptive qualitative approach with, multiple-case study, the results show that the effective leader is one who gets to move the group to achieve the goals. Among the most salient features are: honesty, empathy, optimism, proactivity and creativity. These features relate to the transformational leadership style necessary in situations associated with the financial crisis impacting the Puerto Rican business.
\end{abstract}

Keywords: leadership, leader's traits, leader's roles, economic crisis, Puerto Rican.

\section{RESUMO}

O objetivo do estudo é identificar as características e funçóes mais prevalecentes nos líderes de empresas de Puerto Rico em tempos de crise financeira. Através de uma abordagem qualitativa descritiva, com desenho de estudo de caso múltiplo, os resultados mostram que o líder eficaz é aquele que consegue mover ao grupo para atingir os objetivos. Entre as características mais importantes são: honestidade, empatia, otimismo, proatividade e criatividade. Estes rasgos referem-se ao estilo

* Puertorriqueña, Ph. D., Escuela de Estudios Profesionales, Universidad del Este, Recinto de Carolina, Puerto Rico. Correspondencia con la autora: zberdecia@suagm.edu.

** Puertorriqueña, Ph. D., Escuela de Psicología, Universidad de Puerto Rico, Recinto de Río Piedras, Puerto Rico. Correspondencia con la autora: profa.joycegonzalez@gmail.com. 
Liderazgo corporativo en tiempos de crisis económica: estudio de casos en empresas puertorriqueñas / Berdecía y González

de liderança transformacional necessário em situaçóes associadas com a crise financeira que impacta as empresas porto-riquenhas.

Palavras-chave: liderança, características do líder, os papéis do líder, crise econômica, Puerto Rico.

\section{Introducción}

La competencia global de los mercados, los rápidos cambios tecnológicos, la desregulación, los equipos virtuales y los movimientos en la fuerza laboral, entre otros, son algunos de los factores asociados al ambiente actual de negocios. Algunos autores seńalan que estos acontecimientos han producido crisis en el sector económico en diferentes sectores de la población (Kaufmann, Vrontis, Czinkota y Hadiono, 2012; Pardey, 2007). Como respuesta a los cambios y las crisis, las empresas se han visto obligadas a transformarse para ser cada vez más proactivas y competitivas. Esto implica la necesidad de que las personas que las conforman asuman rápidamente los nuevos retos, sean más eficientes y capaces de liderar para el logro de los objetivos y metas de la organización (Gandossy, Greenslade y KaoPardey, 2008; Castro, Miquilena y Peley, 2006).

Aunque el logro de los objetivos organizacionales depende de una enorme cantidad de factores, la literatura señala que el liderazgo es considerado como uno de los elementos cruciales en la implementación e inicio de cualquier transformación (Chan y Drasgrow, 2001; Carss, 2010), además de que resulta decisivo para alcanzar resultados extraordinarios (Bass, 1985). Por otro lado, el cambio hacia un nuevo paradigma, en el que refleja el paso de un planteamiento administrativo racional tradicional, que recalca la estabilidad y el control, a uno que valora el mismo. La atribución de facultades y las relaciones, requieren que los administradores también sean líderes eficaces frente a los fenómenos sociales, económicos y tecnológicos que se proyectan (Gandossy et al., 2008; Daft, 2008; Spector, 2000).

\section{Revisión de literatura}

Tradicionalmente, se define un líder como la persona a cargo de influir en las actitudes, ideas, sentimientos y conductas de otras personas (Daft, 2008; Spector, 2000). Rost y Baker (2000) definen liderazgo como una relación de influencia que ocurre entre los líderes y sus seguidores, mediante el cual las dos partes pretenden llegar a cambios y resultados reales que reflejen los propósitos que comparten (p. 14). Por otro lado, Castro et al. (2006:83) lo definen como "un fenómeno de interacción social que se manifiesta en todos los grupos de cualquier organización, en el cual el líder eficiente es aquél que logra resultados exitosos al dirigir a los seguidores". Ambas definiciones señalan varios 
elementos básicos del liderazgo, entre los que se destacan los siguientes: a) implica influencia, lo que significa que la relación entre las partes no es pasiva ni coercitiva, y; b) tienen la intención de realizar cambios, y éstos reflejan los propósitos compartidos entre los líderes y sus seguidores con el logro de las metas de la organización. Esto concuerda con los resultados de varios estudios con enfoques cuantitativos en los que muestran que existe una relación positiva entre ciertos tipos de liderazgo y los resultados organizacionales (Bass, 1999; Gardner y Avolio, 1998; Howell y Costley, 2001; Sosik, Potosky y Jung, 2002; Walumbwa, Wang, Lawler y Shi, 2004; Bass y Riggio, 2006; Pedraja, Rodríguez y Rodríguez, 2008; Gandossy et al., 2008; Kaufmann et al., 2012; Darvish y Pour, 2013).

A pesar de las contribuciones intelectuales, al momento del estudio no encontramos estudios cualitativos ni conceptualización que analice con profundidad cómo los rasgos y roles de los líderes están asociados al éxito de la empresa en tiempos de crisis económica. La mayoría de los estudios previos sobre este tema en Puerto Rico han estado enfocados en evaluar los elementos del liderazgo de forma cuantitativa y no en analizar la posible relación de forma inductiva, descriptiva y cualitativa de los rasgos y roles de los líderes en momentos de crisis económica.

El término crisis económica hace referencia a la percepción de la situación financiera mundial que se inició, desde el 2008, en los Estados Unidos. Entre los principales factores causantes de la crisis están: los altos precios de las materias primas, amenazas a las fuentes alimentarias mundiales, una elevada inflación planetaria y la amenaza de una recesión en todo el mundo; así como una crisis crediticia, hipotecaria y de confianza en los mercados. El mundo en general ha caído en una profunda recesión; esta a su vez se define como dos trimestres consecutivos de decrecimiento económico. Según algunas fuentes, se esperaba que la crisis pudiera finalizar en el 2010 pero aún sigue vigente (http://www.buenastareas.com/ensayos/Crisis-Economica-Mundial-2008/1295979. html; Naciones Unidas, 2014).

Puerto Rico no ha sido la excepción en afrontar crisis económica tanto en el sector gubernamental como privado. Por ejemplo, los empresarios en nuestro país han liderado con la quiebra de cuatro instituciones bancarias que resultaron en la desaparición de $\$ 12$ mil millones de dólares de activos; al incremento del déficit comercial de los Estados Unidos con China, según el Instituto de Política Económica, que ha conllevado la pérdida de sobre 20.000 puestos de trabajo. Desde la perspectiva de recursos humanos, la situación financiera en Puerto Rico ha tenido consecuencias en: la disminución de la fuerza laboral, un congelamiento de los programas de recompensas y beneficios otorgados a los empleados y en muchos casos el despido de empleados. Por ejemplo, la tasa laboral en el año 2010 se redujo en un 35\%, una de las tasas más bajas en veinte 
Liderazgo corporativo en tiempos de crisis económica: estudio de casos en empresas puertorriqueñas / Berdecía y González

años, mientras la fuerza laboral en el 2014 se ha disminuido con un total de 987.000 puestos de trabajo (Departamento del Trabajo y Recursos Humanos - Estado Libre Asociado de Puerto Rico, 2014).

Anzorena (s. f.) señala que "en los momentos de crisis e incertidumbre, cuando los manuales para lograr el éxito demuestran su volatilidad, cuando los caminos conocidos no necesariamente conducen al destino deseado y nuestras brújulas nos indican que no siempre hay que ir al norte, emerge con claridad y nitidez la importancia del liderazgo". Por tal motivo, las tareas de los líderes radican en facilitar la adaptación (desarrollar nuevas prácticas, no sólo implementar las mejores), aceptar el desequilibrio (inducir cambios) y generar más liderazgo (dar oportunidades a los colaboradores de liderar proyectos)" (Montelli, 2009, citado en De Bella, 2009). "Entre otras prácticas, Hadden (2009, citado en De Bella, 2009) recomendó llevar a cabo encuestas a los empleados para dirigir las estrategias de recursos humanos, definir claramente la misión de la compañía y asegurarse de que todos la comprendan, desarrollar la interacción y conciencia social, eliminar políticas y procedimientos innecesarios, mantener las acciones de capacitación del personal a pesar de la crisis, generar esquemas de trabajo más flexibles, comunicar con transparencia y pasar más tiempo con los empleados".

Hoy día, para enfrentar los grandes retos globales y económicos, las empresas necesitan de ejecutivos y gerentes con ciertos rasgos de liderazgo entre los que debe estar incluida la adaptación a los cambios y a las limitaciones que impone a sus empresas una economía en recesión. Si integramos al estudio de las empresas nacionales estadounidenses exitosas elementos básicos del liderazgo mencionado por Rost et al. (2000) y Castro et al. (2006) podríamos contestar las siguientes preguntas: ¿qué rasgos de liderazgo prevalecen más en los ejecutivos y gerentes de empresas nativas exitosas? y ¿cuáles son los roles que suelen asumir estos ejecutivos en tiempos de crisis económica? Este artículo aborda estas preguntas mediante los hallazgos de grupos focales realizados a ejecutivos y gerentes de tres empresas nacionales reconocidas como entre los Mejores Patronos del país, según los estudios auspiciados por la empresa Aon Puerto Rico.

\section{Metodología}

El enfoque metodológico utilizado es uno cualitativo descriptivo con diseño de estudio de caso múltiple para obtener una percepción más compleja y profunda del tema de estudio. El uso de esta metodología permitió el estudio intensivo y profundo de diversos aspectos de un mismo fenómeno (Lucca y Berríos, 2009). Los participantes del estudio son ejecutivos y gerentes de tres empresas puertorriqueñas. La selección de estas tres empresas permitió a los investigadores diversas perspectivas para explorar y documentar una unidad de análisis manejable que describa los rasgos y roles más predominantes en 
empresas exitosas en tiempos de crisis económica. La composición sociodemográfica de los participantes del estudio es la siguiente: a) Género: cinco de los participantes son varones y cinco féminas; b) Edad: tres se encuentran entre el grupo de 30 a 39 años de edad, seis entre 40 a 49 años de edad, y uno en la categoría de 50 a 59 años de edad; c) Nivel académico: cinco poseen Bachillerato (4 Administración de Empresas; 1 Ciencias Sociales), cuatro poseen Maestría en Administración de Empresas y uno posee Juris Doctor; d) Puesto: dos son Presidentes, uno es Vice-Presidente, dos son Gerentes de Operaciones y cinco Gerentes de Recursos Humanos; e) Años de experiencia en el puesto: seis poseen entre 5 a 10 ańos de experiencia, dos poseen entre 11 a 15 años y dos sobre 20 años de experiencia en el puesto.

Para la recopilación de datos se utilizó la técnica cualitativa de grupo focal (Lucca et al., 2009). Se organizaron tres grupos focales constituidos por ejecutivos y gerentes de las tres empresas participantes, con el propósito de obtener una amplia gama de información y poder constatarla. El proceso se estableció de manera sistemática para poder categorizar los hallazgos y responder a las preguntas de investigación. Se utilizaron múltiples fuentes para la recopilación de la información, para triangular la misma y cumplir con el criterio de totalidad o visión holística que se contempla en un estudio de caso múltiple (Lucca et al., 2009). Las fuentes para la recopilación de los datos fueron: la revisión de literatura, el análisis de documentos y la entrevista semi-estructurada de un grupo focal influida por las experiencias y opiniones de los participantes.

Los datos de las entrevistas y documentos fueron analizados mediante el modelo de análisis cualitativo de Harry F. Wolcott (1994, citado en Lucca et al., 2009). "Este modelo consta de tres componentes principales: la descripción, el análisis y la interpretación" (p. 484). La técnica analítica general fue leer primero los datos de los temas que surgen de inducción en relación con las áreas claves examinadas en el estudio. Las definiciones de liderazgo, los rasgos y roles de liderazgo fueron algunas de las áreas claves examinadas. Como resultado, revisamos los datos mediante códigos deductivos basados en la literatura relacionada con el liderazgo, sus rasgos y roles en tiempos de crisis económica. Ejemplos de los códigos deductivos incluyen: eficiente logro de resultados, al dirigir seguidores, relación de influencia con propósito compartido, pretende y logra cambios y otros rasgos, al igual que rol del líder en tiempos de crisis.

\section{Resultados}

Para contestar las preguntas de investigación se desarrollaron varias categorías respecto a los hallazgos de los grupos focales, estas son: conceptualización y rasgos de un líder, rasgos presentes en los ejecutivos entrevistados, al igual que el rol del líder en tiempos 
Liderazgo corporativo en tiempos de crisis económica: estudio de casos en empresas puertorriqueñas / Berdecía y González

de precariedad económica. A continuación presentaremos las respuestas de los ejecutivos de cada compañía en las categorías antes mencionadas desde la literatura.

La tabla 1 resume las categorías y los rasgos identificados en relación con ellas en la conceptualización que realizaron los y las participantes respecto a lo que entienden como un(a) líder. La tabla está seguida por citas representativas de uno o más rasgos de estas conceptualizaciones. Además, describe las respuestas ofrecidas por las personas entrevistadas al solicitarle expusieran su definición de un/a líder.

Tabla 1. Conceptualización de líder desde la noción de rasgos.

\begin{tabular}{|c|c|c|c|c|}
\hline Categorías & $\begin{array}{c}\text { Eficiente logro } \\
\text { de resultados al } \\
\text { dirigir seguidores. }\end{array}$ & $\begin{array}{c}\text { Relación de } \\
\text { influencia } \\
\text { con propósito } \\
\text { compartido. }\end{array}$ & $\begin{array}{c}\text { Pretende } \\
\text { y logra } \\
\text { cambios. }\end{array}$ & Otros rasgos. \\
\hline Rasgos & $\begin{array}{c}\text { Logra resultados. } \\
\text { Tiene seguidores. } \\
\text { Da dirección. } \\
\text { Desarrolla a } \\
\text { través de la } \\
\text { participación. }\end{array}$ & $\begin{array}{c}\text { Es modelo. } \\
\text { Proyecta } \\
\text { seguridad. } \\
\text { Es confiable y } \\
\text { honesto/a. } \\
\text { Sus metas y } \\
\text { objetivos son } \\
\text { comunes al } \\
\text { grupo. }\end{array}$ & $\begin{array}{c}\text { Facilita. } \\
\text { Innova. }\end{array}$ & $\begin{array}{c}\text { Demuestra pasión en lo que } \\
\text { hace. }\end{array}$ \\
& & & $\begin{array}{c}\text { Buenas destrezas de } \\
\text { comunicación. } \\
\text { Posee competencia técnica } \\
\text { y se mantiene al día en } \\
\text { temas relacionados a su rol y } \\
\text { funciones. }\end{array}$ \\
& & & $\begin{array}{c}\text { Disciplina para corregir. } \\
\text { Demuestra valentía, } \\
\text { humildad y firmeza. }\end{array}$ \\
\hline
\end{tabular}

Bajo la categoría eficiente logro de resultados al dirigir seguidores, destacamos las siguientes citas:

"...aquella persona que hace que las cosas ocurran o ayuda a otros para que las cosas ocurran. Por lo regular, un líder tiene seguidores, este, tiene gente que lo apoya" (Compañía $\mathrm{A})$.

"Es una persona que lleva su equipo de trabajo a lograr las metas dándole dirección y participación en los procesos y en las decisiones que se vayan a tomar" (Compañía B).

"Darle a la persona su espacio, una persona que vaya desarrollándose en su propio rol para que sea más efectivo” (Compañía C).

Estas son algunas citas que reflejan la categoría relación de influencia con propósito compartido:

"Yo creo que un líder es una persona que es seguido por el grupo que dirige no 
porque lo hayan puesto en la posición sino porque inspira esa confianza y ese respeto" (Compañía C).

"Es una persona que puede lograr llevar a un grupo de personas a un fin común o a unos objetivos comunes en una organización o un grupo" (Compañía B).

"O sea, si la gente entiende lo que tú estás viendo y hacia dónde tú quieres llegar, ellos entonces pueden comprarlo, estar de acuerdo y montarse en el bote y ahí empiezan todos a remar, pero si no saben ni pa' dónde vamos si no lo puedes comunicar" (Compañía B).

"Un líder es una persona que irradia unos rasgos de seguridad en lo que va a ejecutar que hace que un grupo de personas le siga. Y tiene un tal alto grado de confiabilidad también para un grupo, que modela, que las cosas que realiza ese grupo las tome a bien y las siga” (Compañía A).

Entre las citas categorizadas como pretende hacer cambios encontramos: "Líder es aquel que va a facilitar que los procesos se den" (Compañía A).

La tabla 2 resume los rasgos de liderazgo identificados en los ejecutivos entrevistados cuando respondieron: ¿qué rasgos entiende usted que lo distinguen a usted como líder? ¿Qué rasgos usted posee que usted entiende que lo distinguen como un líder? y otros desprendidos de la narrativa de los participantes durante el restante de la entrevista.

Tabla 2. Rasgos de liderazgo presentes en ejecutivos entrevistados.

\begin{tabular}{|c|c|}
\hline Categorías & Hallazgos \\
\hline $\begin{array}{c}\text { Efciente logro de resultados, } \\
\text { al dirigir seguidores. }\end{array}$ & $\begin{array}{c}\text { Participativos: comunican abiertamente y escuchan. } \\
\text { Fomentan y reconocen aportaciones y talentos de otros líderes y } \\
\text { de empleados. }\end{array}$ \\
& Procuran el contacto con empleados. \\
Anticipan y planifican. \\
Accesibles.
\end{tabular}


Liderazgo corporativo en tiempos de crisis económica: estudio de casos en empresas puertorriqueñas / Berdecía y González

\begin{tabular}{|c|c|}
\hline Categorías & Hallazgos \\
\hline Otros rasgos. & $\begin{array}{l}\text { Demuestran pasión en lo que hacen. } \\
\text { Poseen competencia técnica y se mantienen al día en temas } \\
\text { relacionados a su rol y funciones. } \\
\text { Asertivos. } \\
\text { Optimistas. } \\
\text { Reconocen que tienen empleados que pueden saber más que } \\
\text { ellos. } \\
\text { Casual e informal. } \\
\text { Cumplen acuerdos y promesas. } \\
\text { Conciencia del impacto de su rol. }\end{array}$ \\
\hline
\end{tabular}

Los siguientes párrafos presentan varias citas bajo la categoría eficiente logro de resultados, al dirigir seguidores. De la Compañía A destacamos las siguientes citas, las cuales reflejan lo participativo:

"Pero siempre se presentan los resultados. Ellos saben todo el tiempo en dónde están y por dónde van y qué les falta para llegar. O sea, la información se comparte en todos los niveles". "Pero eso ha permitido que el empleado se sienta que es escuchado, en este caso por el dueńo y presidente".

Esta cita refleja el aspecto participativo de la Compañía B:

"Que entiendo que nosotros lo hacemos mucho, y es que con una completa transparencia y una comunicación abierta a todo el mundo, ya sea en reuniones anuales, en reuniones con grupos, se habla de todas las políticas, de todos los procedimientos y del porqué de las cosas".

De la Compañía C:

"Permitir la participación y generación de ideas de ese grupo de personas que se reporta a mí para poder entonces lograr ese objetivo departamental que, de alguna manera, apoyan las estrategias empresariales". "Me gusta también, darle la participación a ellos, que me brinden sus ideas para ver cómo compaginamos, cómo hacemos la idea del grupo la que va a ser exitosa para realizarlo.

De la Compañía A, cómo anticipan, planifican y aportan a la vida de sus seguidores:

"Entendieron que también venía un proceso, un proceso económico, pues ya uno lo venía venir, pero de qué manera podíamos ayudar a que esas economías personales de cada uno de los individuos que trabaja con nosotros no se cayera".

La Compañía C también demostró rasgos de anticipar y planificar. 
"Es importante estar preparado, uno prepararse y demostrarle a los empleados que hay situaciones difíciles, pero juntos podemos lograrlo".

Como parte de la categoría relación de influencia con propósito compartido en la Compañía $\mathrm{C}$, se observó el disfrute por compartir los objetivos:

"Me gusta comunicar con mis compañeros de trabajo cuáles son mis objetivos, cuáles son mis ideas".

En la Compañía A, se encontraron citas con énfasis en cómo se involucran y adaptan a sus empleados:

"Entiendo que son características bien importantes porque, porque si tú te quedas obviamente en un escritorio y no sales y no conoces lo que está pasando en la base, pues tener la capacidad de poder hacer múltiples tareas simultáneamente, pero sin perder de perspectiva adónde quieres llegar, entiendo son cosas bien importantes en un líder". "Entonces, para tú poder llegar a ese grupo, uno ir con una corbata e ir con manga larga, uno pone una pared, entonces, al haber esta informalidad, ellos se sienten parte del grupo. En parte, se sienten aceptados y pueden llegar a uno de una manera más directa, sin tanto protocolo".

La compañía B también compartió el asunto de involucrarse con proyectos y empleados:

"Y características que tengo diferente, yo soy el tipo de líder que me enrollo las mangas y me meto con los muchachos a hacer el trabajo y liderar, con el ejemplo, en todos los aspectos". "El mantener contacto con los empleados, ir allá, si ven cosas las discutimos y tratamos de manejarlo, lo hablamos, nos reunimos, yo creo que en parte eso nos ha ayudado".

La Companía C también lo demuestra:

"Me gusta enrollarme las mangas y trabajar con ellos, estar presente con ellos, envolverme en los asuntos que son críticos y me gusta compartir".

La siguiente cita de la Compañía $\mathrm{C}$ demuestra el rasgo de modelar valores y estilos que desean fomentar:

"Me gusta dar el ejemplo; un ejemplo de compromiso, de que las cosas hay que hacerlas dentro del tiempo y tratar de ayudarlos para que se consigan los objetivos".

En la Compañía A facilitan y enseñan, como parte de la categoría de pretender/lograr cambios:

"Yo le cuento anécdotas personales, que por ejemplo, la primera vez que yo me senté frente a una computadora, yo decía el poder humano y la mentalidad 
Liderazgo corporativo en tiempos de crisis económica: estudio de casos en empresas puertorriqueñas / Berdecía y González

humana es más grande que una máquina, así que yo ya la puedo dominar a ella y no ella a mí, y ahí es cómo perderle el miedo, y entonces cómo llevarlos a que le pierdan el miedo y comiencen a usarlo". "Claro está, pero de la mano, porque nos convertimos en sus facilitadores para que el proceso se dé”.

Entre la categoría otros rasgos está el cumplir promesas y la conciencia del impacto de su rol:

"Cumplimos con nuestras promesas, si esto fue lo que se acordó esto es lo que se cumple” (Compañía A).

"Cualquier cosa que yo diga dentro de la empresa ocasiona alegría, tristeza o preocupación: puedo afectar el sueńo de una persona, puedo afectar su estado de ánimo, puedo afectar hasta su matrimonio solamente con un comentario o una opinión". "Yo no tengo opinión, ni ella ni ella tienen opinión porque lo que ellas digan es ley para mucha gente y afecta a esas personas". "Y basado en esa responsabilidad que uno tiene, puede ser cualquiera, pero lo que uno dice afecta la vida de otra persona” (Compañía A).

"En mi caso, siempre lo hablábamos, cuando vayas a una planta, dar una sonrisita siempre, porque ellos están pendientes a todos tus movimientos y escuchan todo, así que eso es importante" (Compañía A).

Entre los otros rasgos encontramos competencia técnica y humildad. Las siguientes citas de la Compañía B las demuestran respectivamente:

"Saber mucho del tema hace que otros quieran seguirte y te respetan porque saben que como "pares" del contenido técnico, él sabe". "Muchas veces pueden saber mucho más, técnicamente, que tú de ese tema”.

La tabla 3 resume el rol que asumieron los ejecutivos de las tres empresas, durante tiempos de crisis económica. En la primera columna están los hallazgos y en la segunda, ejemplos de citas para elaborar cada hallazgo. 
Tabla 3. Rol del líder en tiempos de crisis.

\begin{tabular}{|c|c|}
\hline Hallazgos & Citas \\
\hline $\begin{array}{l}\text { Comunicar abierta } \\
\text { y constantemente la } \\
\text { situación económica } \\
\text { de la compañía y } \\
\text { razonamiento de las } \\
\text { decisiones tomadas. }\end{array}$ & $\begin{array}{l}\text { "Bueno, por ejemplo, ahora mismo estamos trabajando una } \\
\text { estrategia de comunicar a las personas que (Compañía B) está } \\
\text { bien, está mejorando". "Obviamente, pasando las crisis que han } \\
\text { pasado en los últimos dos años y estamos buscando la manera } \\
\text { de decirle a nuestros empleados que vamos bien". "Y si algo está } \\
\text { mal, pues uno también lo puede decir: "mira, este cliente está } \\
\text { cerrando pero tenemos esperanza con este otro, y que sepan lo } \\
\text { que está ocurriendo". "Yo creo que lo más que desespera es el } \\
\text { desconocimiento". "Si yo te digo que el cliente principal está } \\
\text { cerrando proyectos, pero te lo digo yo y te comunico lo que estamos } \\
\text { haciendo, van a estar mucho más tranquilos a que no sepa nada". } \\
\text { "Mira este ańo perdimos (X) millones, pues perdimos (X)... mira, se } \\
\text { lo decimos". Compańía C. } \\
\text { "Como les digo, todos los días se sabe dónde estamos en números y } \\
\text { todos los empleados se enteran". Compańía A. }\end{array}$ \\
\hline $\begin{array}{c}\text { Tomar decisiones } \\
\text { administrativas } \\
\text { considerando un balance } \\
\text { entre la necesidad } \\
\text { económica y los } \\
\text { intereses, necesidades y } \\
\text { preocupaciones de los } \\
\text { empleados. }\end{array}$ & $\begin{array}{c}\text { "Vemos que hay una crisis, nos sentamos primero los directores } \\
\text { a desarrollar esa infraestructura y la empezamos a comunicar } \\
\text { completamente". Compañía B. }\end{array}$ \\
\hline $\begin{array}{l}\text { Usar anécdotas } \\
\text { personales y analogías } \\
\text { para comunicar } \\
\text { mensajes que incluyan } \\
\text { balance de la realidad y } \\
\quad \text { optimismo. }\end{array}$ & $\begin{array}{l}\text { "Vamos todos juntos a remar y nosotros vamos a estar al frente } \\
\text { diciendo si vamos bien o vamos mal". Compañía B. }\end{array}$ \\
\hline Acercarse a empleados. & $\begin{array}{l}\text { "En el caso mío, estar con los empleados de base y con los recursos". } \\
\text { "Están las cosas difíciles, tenemos que buscar otras alternativas, } \\
\text { otros clientes, en vez de ir a otros lugares, pero yo no puedo dejar } \\
\text { de visitar o darme la vuelta en las áreas de trabajo". "En el tipo de } \\
\text { compañía que somos, parte cultural, somos de los que estamos ahí } \\
\text { con ellos". Compañía B. }\end{array}$ \\
\hline Ofrecer dirección. & $\begin{array}{l}\text { "Vamos todos juntos a remar y nosotros vamos a estar al frente, } \\
\text { diciendo si vamos bien o vamos mal". Compañía B. }\end{array}$ \\
\hline
\end{tabular}


Liderazgo corporativo en tiempos de crisis económica: estudio de casos en empresas puertorriqueñas / Berdecía y González

\begin{tabular}{|c|c|}
\hline $\begin{array}{l}\text { Identificar nuevas } \\
\text { formas de realizar } \\
\text { y lograr resultados } \\
\text { / enfoque en } \\
\text { oportunidades. }\end{array}$ & $\begin{array}{l}\text { "Tenemos que estar en actitud positiva, reinventando, reaprendiendo } \\
\text { nuevamente, buscando nuevas ideas, inventando, qué podemos } \\
\text { hacer, cuál es la combinación de productos más atractivos para el } \\
\text { público para seguir adelante". Compañía A. } \\
\text { "Nos hemos enfocado en las oportunidades, qué podemos ver } \\
\text { dentro del proceso y no enfocarnos en lo que es la dificultad de la } \\
\text { crisis económica”. Compañía A. }\end{array}$ \\
\hline $\begin{array}{l}\text { Mantener imagen de } \\
\text { la compañía, velar por } \\
\text { buenas relaciones con } \\
\text { clientes y calidad de } \\
\text { productos/servicios. }\end{array}$ & $\begin{array}{l}\text { "Vamos para delante, esto va bien, remodelamos la tienda en la } \\
\text { medida que se puede, se le cambia la imagen, se le envían rótulos } \\
\text { nuevos, uniformes nuevos, fue un gran impacto también". "Esa } \\
\text { campańa de nuevos uniformes gustó mucho". Compañía A. } \\
\text { "Pagar a tiempo el crédito". "Eso es tu confianza, eso es tu nivel de } \\
\text { confianza". "Número uno, si tú no tienes un nivel de confianza con tus } \\
\text { suplidores ni con tus empleados, tú lo perdiste todo". Compañía A. }\end{array}$ \\
\hline $\begin{array}{l}\text { Involucrarse en las tareas } \\
\text { de los empleados. }\end{array}$ & $\begin{array}{l}\text { "Lo que pasa es que él está en todas las bases". "El presidente es una } \\
\text { persona que está en todas las bases". Compañía A. }\end{array}$ \\
\hline $\begin{array}{l}\text { Reconocer ajustes } \\
\text { que deben realizar en } \\
\text { términos de estilos } \\
\text { gerenciales, en lo } \\
\text { personal y en mensajes } \\
\text { que comunican. }\end{array}$ & $\begin{array}{l}\text { "Obviamente, yo cogí el cantazo económicamente primero que } \\
\text { nadie y todos tenían que saber que si yo soy el líder yo tenía que } \\
\text { dar el ejemplo". "Todavía tengo el mismo carro desde hace } 13 \text { ańos, } \\
\text { no hay lancha, no hay casa de playa, yo se los cantaba así mismo". } \\
\text { Compañía B. } \\
\text { "Nosotros enlistamos todo; plan médico era el 100\%, celular". } \\
\text { "Básicamente listamos en el presupuesto todo lo que tú puedas } \\
\text { eliminar antes de tocar el salario, sabiendo que reducirle el salario } \\
\text { a todos era una opción versus sacar a varios de la plantilla". } \\
\text { Compańía B. }\end{array}$ \\
\hline
\end{tabular}

\section{Conclusiones}

Este trabajo ha tenido como propósito el identificar los rasgos y roles más prevalecientes en los líderes de empresas puertorriqueñas en momentos de crisis financiera. Algunos teóricos sostienen que en momentos de crisis e incertidumbre emerge la importancia del liderazgo para el logro de las metas organizacionales (Montelli, 2009, citado en De Bella, 2009). Los participantes del estudio conceptualizan un liderazgo caracterizado por los rasgos de proveer dirección, facilitar la participación, credibilidad de sus seguidores, disciplina, asertividad y accesibilidad de la información. Dicho de otra manera, la conceptualización vigente es que el líder eficiente es aquel que consigue mover al grupo a la consecución de la meta, al proveer dirección, disciplina, ser participativo, accesible, asertivo y tener credibilidad con sus empleados.

Los resultados confirman estudios previos sobre la importancia del desarrollo de la confianza de los líderes a través de una comunicación abierta, honesta y con un manejo adecuado 
de las expectativas (Hayes y Perry, 2011). Esto se muestra cuando los tres presidentes de las compañías manifestaron entre sus rasgos el comunicar abierta y honestamente a sus empleados situaciones económicas de la empresa, utilizando diversas formas para compartir lo que estaba pasando y mantener expectativas claras del negocio. Por otro lado, Paternotte (2006) sostiene que un líder que ha ganado la confianza de su equipo genera un ambiente positivo en el cual todas las personas pueden expresarse abiertamente y ser escuchadas. Los tres grupos de ejecutivos entrevistados mencionaron rasgos acordes con este atributo, tales como: la empatía, el optimismo y el acceso a la información.

En cuanto a seguridad, los participantes incluyeron definiciones del liderazgo, manifestaron rasgos y ejercieron un rol en tiempos de crisis. Conforme con este atributo, entre ellos: logran resultados, dan dirección, proyectan seguridad, poseen metas y objetivos comunes al grupo, demuestran pasión en lo que hacen, son innovadores, poseen competencia técnica y se mantienen al día en temas relacionados a su rol y funciones. En adición, demostraron la proactividad asociada a realizar los ajustes necesarios para asegurar el futuro de la companía.

Otro rasgo importante y destacado en el estudio fue el de liderazgo empresarial. De acuerdo con Siddiqui (2007), este concepto incluye comportamientos de los líderes en pro de los intereses de la organización para el crecimiento de todas las personas involucradas y el logro del propósito propuesto. El liderazgo empresarial facilitó que estos grupos de ejecutivos pudieran adaptarse a los cambios requeridos por la precariedad económica, mantener la calidad de sus productos o servicios y proteger la mayor cantidad de empleos posible. En otras palabras, lograron el mejor balance entre el capital fiscal y el humano. Demostraron ser excelentes agentes de cambio, con la flexibilidad, innovación y capacidad de transmitir mensajes con visión, sin dejar de incluir a sus equipos en el proceso, por lo que se puede concluir que sus líderes demostraron efectividad empresarial. En conclusión, los resultados expuestos muestran una relación positiva entre ciertos rasgos y roles de los líderes corporativos y su capacidad para sobrevivir y potenciar el crecimiento de sus empresas, aun en tiempos de crisis económica.

\section{Bibliografía}

Anzorena, O. (s. f.). Liderazgo en tiempos de crisis e incertidumbre. Recuperado de: http:// www.sht.com.ar/archivo/liderazgo/lider_crisis.htm.

Bass, B. (1985). Leadership and performance beyond expectations. New York: The Free Press.

Bass, B. (1999). Two decades of research and development in transformational leadership. European Journal of Work and Organizational Psychology, 8(1), 9-13.

Bass, B. M. \& Riggio, R. E. (2006). Transformational leadership. Mahwah, NJ: Erlbaum. 
Liderazgo corporativo en tiempos de crisis económica: estudio de casos en empresas puertorriqueñas / Berdecía y González

Carss, B. W. (2010). The measurement of transformational leadership skills in the workplace: the path towards innovative and high performance teams. Recuperado de: http://www.ufv.ca/Assets/Writing+Centre/The+Measurement+of+Tran sformational+Leadership+Skills+in+the+Workplace+The+Path+towards+In novative+and + High + Performance+Teams.pdf.

Castro, E.; Miquilena, E. \& Peley, R. (2006). Las nuevas tendencias del liderazgo: hacia una nueva visión de las organizaciones educativas. Omnia 12(1), 83-96.

Chan, K. Y. y Drasgrow, F. (2001). Toward a theory of individual differences and leadership: understanding the motivation to lead. Journal of Applied Psychology, 83 (3), 481-498.

BuenasTareas.com (2012). Crisis Económica Mundial 2008. Recuperado de http://www. buenastareas.com/ensayos/Crisis-Economica-Mundial-2008/1295979. html.

Daft, R. L. (2008). The leadership experience, Fourth Edition. USA, South-Western Cengage Learning.

Darvish, H. y Pour, M. S. (2013). Measuring employees job satisfaction as outcomes of Multifactor Leadership Questionnaire (MLQ) leadership styles: an empirical study. Reef Resources Assessment and Management Technical Paper, 38(2), 622-627.

De Bella, D.; Montelli, A. \& Hadden, R. (2009). Las nuevas prácticas del liderazgo en tiempos de crisis. Human Capital Forum, 5ta. Edición. Recuperado de http://www.materiabiz.com/mbz/capitalhumano/ vsp?nid=43148.

Departamento del Trabajo y Recursos Humanos - DTRH. Estado Libre Asociado de Puerto Rico (2015). Estadísticas de Empleo y Desempleo Diciembre 2014. Negociado de Estadísticas del Trabajo. Recuperado de: http://www.trabajo.pr.gov.pdf/Estadisticas/2014/GT/gt12.pdf.

Gandossy, B.; Greenslade, S. y KaoPardey, T. (2008). Managing leadership in turbulent times - why and how the global top companies for leaders optimize leadership talent in emerging markets. White Paper. Recuperado de:

http://www.aon.com/attachments/thought-leadership/Managing_Leadeship_Turbulent_Times_033009.pdf.

Gardner, W. L. \& Avolio, B. J. (1998). The charismatic relationship: a dramaturgical perspective. Academy of Management Review, 23, 32-58.

Hayes James, E. \& Perry Wooten, L. (2011). Leadership in turbulent times: competencies for thriving amidst crisis. Social Science Network, 1-26. Recuperado de: http://ssrn.com/abstract=555966. 
Howell, J. P. \& Costley, D. L. (2001). Understanding behavior for effective leadership. USA, Prentice Hall.

Kaufmann, H. R.; Vrontis, D.; Czinkota, M. y Hadiono, A. (2012). Corporate branding and transformational leadership in turbulent times. Journal of Product \& Brand Management, 21(3), 192-204.

Lucca Irizarry, N. \& Berríos Rivera, R. (2009). Investigación cualitativa: fundamentos,

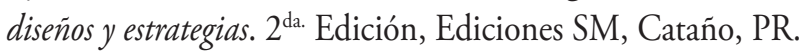

Pardey, David. (2007). "Leadership in turbulent times: Effective leadership during times of organizational change". Strategic HR Review, 6(5), 16-19.

Paternotte, S. (2006). Guiding principles of trusted leadership: leadership built to serve and prevail in times of change. Management - Lex Jansen, 1-14. Recuperado de: http://www.lexjansen.com/pharmasug/2006/Management/MA06.pdf.

Pedraja, L.; Rodríguez, E. \& Rodríguez, J. (2008). Importancia de los estilos de liderazgo sobre la eficacia: un estudio comparativo entre grandes y pequeñas y medianas empresas privadas. Revista de Ciencias Sociales, XIV (1), 20-29.

Rost, J. C. y Baker, R. A. (2000). Leadership education in colleges: toward a $21^{\text {st }}$ century paradigm. The Journal of Leadership Studies, 7(1), 3-12.

Siddiqui, S. (2007). An empirical study of traits determining entrepreneurial leadershipan educational perspective. Skyline Business Journal, IV (1), 37-44.

Naciones Unidas (2014). Situación y perspectivas de la economía mundial 2014. New York. Recuperado de:

http://www.un.org/en/development/desa/policy/wesp/wesp_current/ 2014wesp_es_sp.pdf.

Sosik, J.; Potosky, D. \& Jung, D. (2002). Adaptive self-regulation: meeting others expectations of leadership and performance. The Journal of Social Psychology, 142(2), 211-232.

Spector, P. (2000). Psicología industrial y organizacional: investigación y práctica. Manual Moderno, México.

Walumbwa, F; Wang, P.; Lawler, J. \& Shi, K. (2004). The role of collective efficacy in the relations between transformational leadership and work outcomes. Journal of Occupational and Organizational Psychology, 77, 551-530.

Zhu, W.; Chew, I. y Spangle, W. (2005). CEO transformational leadership and organizational outcomes: the mediating role of human-capital-enhancing human resource management. Leadership Quarterly, 16, 39-52. 
Liderazgo corporativo en tiempos de crisis económica: estudio de casos en empresas puertorriqueñas / Berdecía y González

\section{Cómo citar este artículo:}

Berdecía, Z. y González, J. (2016). "Liderazgo corporativo en tiempos de crisis económica: estudio de casos en empresas puertorriqueñas", Oikos No 41, 33-48, Escuela de Administración y Economía, Universidad Católica Silva Henríquez (UCSH), Santiago de Chile.

[http://ediciones.ucsh.cl/revistas.php]

Fecha de recepción: 30/03/2016

Fecha de aceptación: 04/05/2016 